\title{
Discriminative ability of quality of life measures in multiple sclerosis
}

\author{
Kirsten M. Fiest ${ }^{1}$, Jamie Greenfield ${ }^{2,3}$, Luanne M. Metz ${ }^{2,3}$, Scott B. Patten ${ }^{3,4,5,6}$, Nathalie Jetté $2,3,4,7$ \\ and Ruth Ann Marrie ${ }^{1,8^{*}}$
}

\begin{abstract}
Background: Though many people with multiple sclerosis (MS) have comorbidities, the use of generic and disease-specific health related quality of life (HRQOL) scales to discriminate the effects of comorbidity has not been established. The utility of these scales to discriminate differences between persons with varying levels of disability is also unknown.
\end{abstract}

Methods: Using online questionnaires, a convenience sample of Albertans with MS was recruited between July 2011 and March 2013. Participants completed demographic questions, a validated comorbidity questionnaire, a self-reported disability scale, and the following HRQOL scales: the Short Form (SF)-36, SF-6D, Health Utilities Index-Mark III (HUI-III), and Multiple Sclerosis Quality of Life-54 (MSQOL-54). The ability of each HRQOL scale to distinguish between comorbidity groups was assessed using a one-way analysis of covariance, adjusting for age, sex, disease course, and disability level.

Results: Five hundred sixty three participants completed all relevant questionnaires. All HRQOL measures distinguished between persons with or without depression, while none were able to distinguish between participants with or without hypertension, thyroid disease, irritable bowel syndrome, or osteoporosis. The SF-36 physical scale, SF-6D, HUI-III, and MSQOL-54 physical scales were able to distinguish between all disability groups, though the HUI-III was better able to distinguish between individuals with moderate versus severe disability.

Conclusions: Disease-specific measures would discriminate better between those with and without comorbidities than generic-specific measures and the HUI-III would discriminate best between persons with differing severities of disability. Generic or disease-specific measures may be useful in future studies examining the effects of comorbidity in MS and the effects of treatment of comorbidities in MS.

Keywords: Multiple sclerosis, Quality of life, Health utility, Comorbidity, Concordance

\section{Background}

Comorbidity is increasingly recognized to be common in multiple sclerosis (MS), including physical and psychiatric comorbidity [1]; over $40 \%$ of the MS population may suffer from comorbidities depending on the age and sex of the population [2]. Some studies suggest that the presence of certain comorbidities is associated with delayed diagnosis, accelerated disability progression, and an increased risk of gadolinium-enhancing lesions on MRI [3, 4].

\footnotetext{
* Correspondence: rmarrie@hsc.mb.ca

'Department of Internal Medicine, University of Manitoba, GF533, 820

Sherbrook Street, Winnipeg, MB R3A 1R9, Canada

${ }^{8}$ Department of Community Health Sciences, University of Manitoba,

Winnipeg, MB, Canada

Full list of author information is available at the end of the article
}

Several studies also report that physical and psychiatric comorbidity adversely affect health-related quality of life (HRQOL), suggesting that improved treatment of comorbidity may be one means of improving HRQOL in MS [5-7]. However, prior studies of the association between comorbidity and HRQOL differ with respect to the instruments used to assess HRQOL [8-11]. Instruments used in prior comorbidity studies in MS include the Short Form (SF)-12 [12], SF-36 [9], the Multiple Sclerosis Quality of Life-54 (MSQOL-54) [8] and the Health Utilities Index (HUI) [13], among others.

Previous research suggests that the HUI-III performs better than other health utility measures in discriminating between persons with MS with differing degrees of 
disability, being able to distinguish mild, moderate, severe, and very severely disabled persons [14]. It is unknown which instrument is most sensitive to the effect of comorbidity on HRQOL. This is relevant to future studies evaluating the impact of interventions directed at mitigating the effects of comorbidity on MS, and to studies aimed at evaluating the effects of comorbidity on MS. We aimed to compare the discriminative ability of HRQOL instruments, including the MSQOL-54, SF-36, SF-6D, and the HUI-III, in a prevalent MS population with and without comorbidity. We also examined whether the included measures performed similarly in persons with and without disability. Specifically, we hypothesized that: (1) generic measures would discriminate better between those with and without comorbidities than disease-specific measures; and (2) the HUI-III would discriminate best between persons with differing severities of disability.

\section{Methods}

\section{Study population}

The Alberta Multiple Sclerosis Initiative (TAMSI) was a longitudinal (July 2011-December 2013) observational study that used online questionnaires to collect patientreported information about the safety, experiences, and outcomes following chronic cerebrospinal venous insufficiency (CCSVI) treatment. TAMSI had multiple modules and patients were able to select, based on their own schedules, which modules to complete. TAMSI was also designed to provide information about the health status and living conditions of all Albertans with MS, regardless of CCSVI treatment status.

For the current study, we included participants who completed all of the HRQOL measures (see below) and baseline surveys (demographics, MS characteristics, comorbidity status). To ensure the time period referred to in the questionnaires was consistent, the HRQOL surveys had to be completed within a two-week window. In addition, all baseline surveys had to be completed within 30 days of the HRQOL surveys. If participants filled out questionnaires at multiple time-points, the baseline information was used.

\section{Measures}

Demographic information was collected, including age, sex, race, marital status, employment status, and level of education. Participants also reported characteristics of their MS including the date of onset of MS symptoms, use of disease modifying therapy, and disease course.

\section{Disability}

Disability status was reported by participants using the Patient-Determined Disease Steps (PDDS) [15], a validated self-report measure that strongly correlates with physician-assessed Expanded Disability Status Scale (EDSS) scores [16]. Based on the PDSS score [range 0 (no disability)-8 (bedbound)], disability was categorized into four groups: normal $=0$; mild $=1-2$; moderate $=3-$ 5 ; and severe $=6-8$.

\section{HRQOL measures}

Many measures have been used to quantify HRQOL in MS, including disease-specific and generic measures. Generic measures usually assess physical and mental health, are intended to be applied to any adult population, and are useful for making comparisons across diseases. Disease-specific measures are developed for a specific disease and may be more responsive to change in that specific disease than generic measures [17], but do not support comparisons across diseases, and may not capture the effects of comorbidities. Generic measures can be classified as health profiles or preference-based measures based on an individual preference or value for certain health states (utility measures). Health profile measures produce scores representing different domains of HRQOL, while utility measures result in a single weighted value that represents a person's health state according to their preference. For this study we employed two HRQOL measures: a generic health utility measure, the HUI-III [18] and an MS-specific health profile measure, the MSQOL-54 [19]. From the MSQOL-54, we derived both the SF-36 [20], a generic health profile measure, and the SF-6D [21], a generic health utility measure. See Additional file 1: Table S1 for a comparison of the characteristics of all included HRQOL measures.

Short Form-36

The SF-36 is a 36 -item generic measure of HRQOL that assesses eight domains: vitality, physical functioning, bodily pain, general health perceptions, physical role functioning, emotional role functioning, social role functioning, and mental health. The SF-36 generates two aggregate scores summarizing physical HRQOL (Physical Component Score, PCS-36) and mental HRQOL (Mental Component Score, MCS-36). Scores on the PCS-36 and MCS-36 range from 0 to 100, and are standardized to reflect a general population mean of 50 and standard deviation of 10 . Higher scores indicate better HRQOL. The SF-36 has been extensively validated in MS, and has good reliability and validity [22, 23].

\section{$S F-6 D$}

A health utility measure, the SF-6D is a subset of 11 questions from the SF-36, restructured into six dimensions: social functioning, physical functioning, mental health, pain, role limitations, and vitality. A single summary score is obtained, with possible scores ranging from 0.301 to 1.00 , where 1.00 is perfect health. The validity and reliability of the SF-6D have been established in MS 
[24], and it has shown to distinguish between different levels of disability as measured by the EDSS [14].

\section{HUI-mark III}

The HUI-Mark III (HUI-III) is a 16-item generic multi-attribute utility-weighted measure of HRQOL that assesses eight domains, including hearing, speech, vision, ambulation, emotion, pain, cognition, and dexterity. An overall score of 1.00 on the HUI-III indicates perfect health, while a score of 0.00 is death, and negative scores (up to -0.36) represent health states worse than death. Previous research suggests the HUI-III outperforms other utility measures in MS [14, 24].

\section{MSQOL-54}

The MSQOL-54 is an MS-specific HRQOL measure that supplements the SF-36 with 18 disease-specific questions, measuring cognitive functioning, energy, pain, social functioning, sexual functioning, health anxiety, and overall quality of life. Similarly to the SF-36, two aggregate scores are generated, one for physical HRQOL (PCS-54) and one for mental HRQOL (MCS-54). The standardized scores range from 0 to 100 , with higher scores indicating better HRQOL. The MSQOL-54 has been extensively validated, though the additional benefit of MS-specific items (over the SF-36) has been questioned $[25,26]$. In this study, the sexual functioning subscale was optional, and participants were able to skip those questions if they felt they did not apply. For the present analyses, an alternate scoring system was used to account for the amount of missing items on the sexual functioning subscale (11.4\% in this sample). A modified scoring algorithm was employed where the weight applied to the sexual functioning subscale was evenly distributed among the remaining seven subscales. This modified algorithm was applied to all participants, regardless if they had missing items on the sexual functioning subscale.

\section{Comorbidity}

Participants reported the presence or absence of comorbidities using a modified version of a validated questionnaire, with inclusion of additional comorbidities [27]. The comorbidities examined were: depression, anxiety disorders, bipolar disorder, alcoholism, hyperlipidemia, hypertension, migraine, lung trouble, thyroid disease, irritable bowel syndrome (IBS), osteoporosis, cataracts, diabetes, heart trouble, inflammatory bowel disease (IBD), rheumatoid arthritis, fibromyalgia, epilepsy, glaucoma, diseases of the arteries, lupus, and Parkinson's disease. We analyzed comorbidities in four ways: (1) number of comorbidities (grouped as none, one, two, three or more); (2) presence/absence of each comorbidity; (3) any physical comorbidity (hyperlipidemia, hypertension, migraine, lung trouble, thyroid disease, IBS, osteoporosis, cataracts, diabetes, heart trouble, IBD, rheumatoid arthritis, fibromyalgia, epilepsy, glaucoma, diseases of the arteries, lupus, Parkinson's disease); and (4) any psychiatric comorbidity (depression, anxiety disorders, bipolar disorder).

\section{Data analysis}

Proportions and means (SD) were calculated for categorical and continuous data, respectively. Comorbidities were retained in the multivariate analyses if they had a prevalence of at least $5 \%$ in our sample (depression, hyperlipidemia, hypertension, migraine, lung trouble, thyroid disease, IBS, osteoporosis, anxiety disorders), to ensure enough individuals to provide a stable estimate.

\section{HRQOL measure concordance}

First, we examined the relationships between the HRQOL measures. We produced Spearman correlation matrices to compare correlations between each HRQOL measure. We considered correlations of 0.39 and below to be low, those between 0.40 and 0.59 as moderate, and those 0.60 and above as high [28].

\section{HRQOL discriminant ability}

Second, we examined the ability of each HRQOL measure to distinguish between groups. We did this for persons with different numbers of comorbidities (none, one, two, three or more), the absence/presence of each comorbidity, any physical comorbidity, any psychiatric comorbidity, and disability level (PDDS: normal, mild, moderate, severe). Comorbidity status or disability level were used as the intergroup factor in a one-way analysis of covariance (ANCOVA), with age, sex, disease course, and disability level (when not the intergroup factor) used as covariates. For all analyses the dependent variable was one of the HRQOL measures. Relative efficiency (RE) is a method of assessing the discriminative ability of different instruments to distinguish between groups; in this study we focused on groups according to comorbidity status or disability status. The RE of the instruments was calculated as the ratio of the between group ANCOVA F-statistics; Tukey statistics were used for the pairwise comparisons. [29] The instrument with the largest Fvalue/Tukey-value would have the greatest discriminative ability and was selected as the reference category $(\mathrm{RE}=1)$ to determine the $\mathrm{RE}$ of all other instruments. Values of RE range from 0 to 1 , where 1 indicates no difference in the instrument's discriminative ability compared to the reference instrument. All reported $p$-values from the ANCOVA were corrected for multiple comparisons using Tukey's procedure.

All data were analyzed using STATA version 11.0. 


\section{Results}

Ultimately, 868 participants enrolled in this study, of whom 563 (64.9\%) provided complete data for the HRQOL measures, disability, and comorbidity (Fig. 1) and were the subject of further analyses (Table 1). Their characteristics were similar to those expected for a prevalent MS population [30]. The distribution of comorbidities was as follows: none $(n=197,35.0 \%)$; one ( $n=163,29.0 \%)$; two $(n=107,19.0 \%)$, and three or more $(n=96,17.1 \%)$ comorbidities. Physical comorbidities were present in $54.4 \%(n=306)$ while psychiatric comorbidities were present in $29.1 \%(n=164)$. The most common comorbidities were depression, hypertension, and hyperlipidemia (Table 2).

The mean (SD) score was 38.4 (12.3) for the PCS-36, 48.5 (11.9) for the MCS-36, 0.56 (0.32) for the HUI-III, 0.68 (0.13) for the SF-6D, 57.4 (22.2) for the PCS-54 and 63.2 (19.1) for the MCS-54.

\section{Comorbidity}

Mean HRQOL scores for all scales were highest in those without comorbidities, and decreased with an increasing number of comorbidities (Additional file 2: Figure S1). The ANCOVA revealed statistically significant overall effects of the number of comorbidities for all HRQOL measures (PCS-36 F $=10.70, p<0.0001$; MCS-36 F = 9.28, $p<0.0001$; SF-6D F $=11.56, p<0.0001$, HUI-III F $=9.68, p<0.0001$; PCS $-54 \mathrm{~F}=19.05, p<0.0001$; MCS $54 \mathrm{~F}=13.47, p<0.0001$; Additional file 3: Table S2), indicating that each measure was able to distinguish between people with differing numbers of comorbidities (none, one, two, three or more). Further pairwise comparisons showed that no measure was able to distinguish between persons without comorbidities and persons with one comorbidity or persons with two comorbidities versus three or more. The PCS-36, SF-6D, HUI-III, and PCS-54 were able to distinguish between all other combinations of the number of comorbidities (all $p<0.05$ ), while the MCS-54 was able to distinguish between persons with none or two, none or three or more, and one or three or more comorbidities; and the MCS-36 was only able to distinguish between persons with none or three or more comorbidities. The RE of each instrument by comorbidity status is displayed in Fig. 2 .

All HRQOL measures were able to distinguish between those with and without psychiatric comorbidities (all $p<0.005)$. Neither the MCS measures, nor the HUIIII, were able to distinguish between those with and without physical comorbidities. When each comorbidity was examined individually, all HRQOL measures were able to distinguish between those persons with or without depression (all $p<0.001$ ) (Additional file 3: Table S2). Hyperlipidemia was distinguishable by the PCS-36, PCS-54, and MCS-54; migraine by the PCS-36, SF-6D, and PCS-54; lung trouble by the PCS-36; and anxiety disorders by all except the PCS-36. None of the HRQOL measures were able to distinguish participants with or without hypertension, thyroid disease, IBS, or osteoporosis. Based on the F-test, the PCS-54 performed the best for number of comorbidities, while the PCS-36 performed best for any physical comorbidity and the MCS-54 performed best for any psychiatric comorbidity.

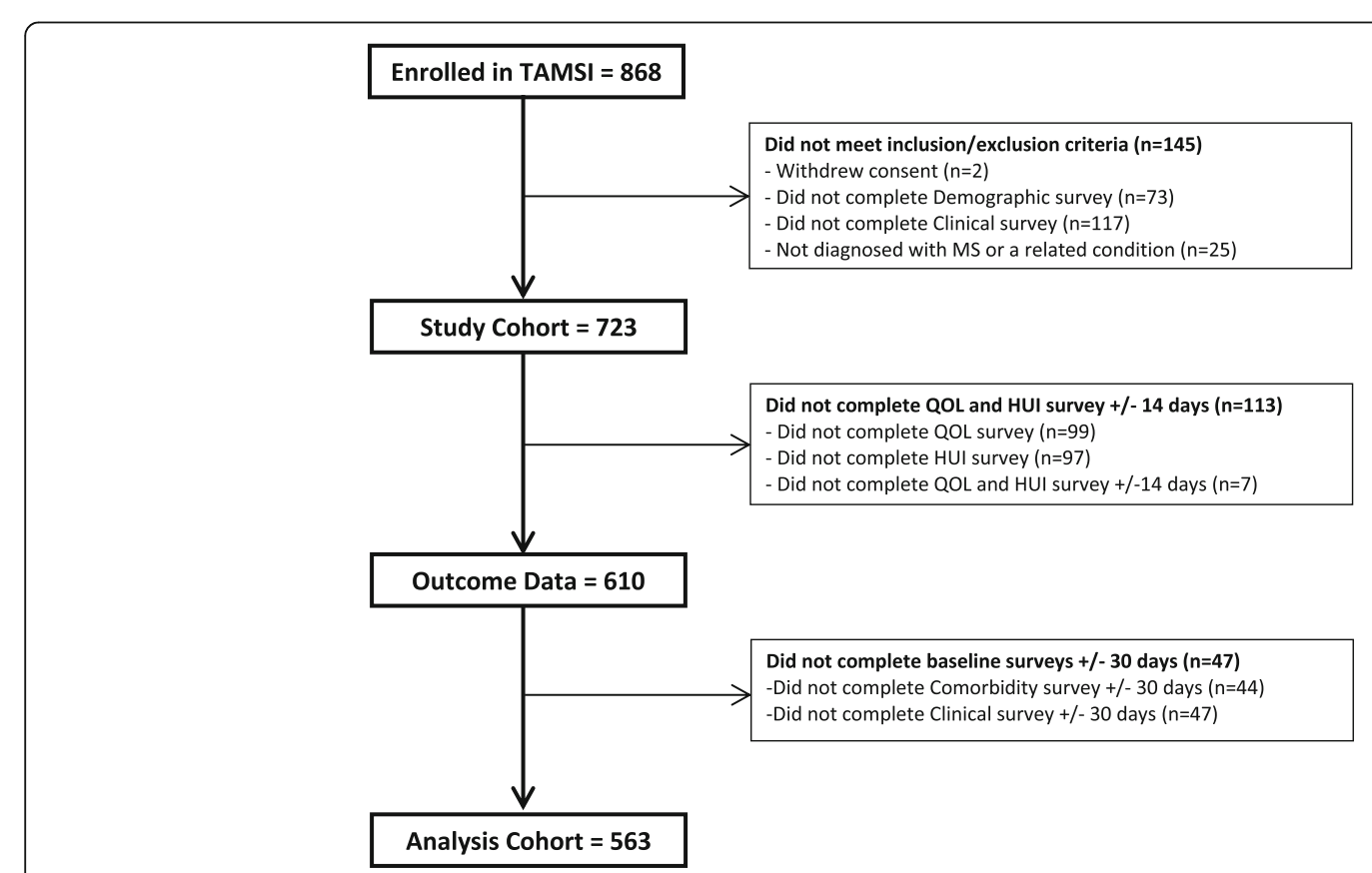

Fig. 1 Participant flow diagram 
Table 1 Demographic and MS characteristics of TAMSI sample

\begin{tabular}{ll}
\hline Variable & Sample $(N=563)$ \\
\hline Age (years), mean (SD) & $47.6(11.1)$ \\
Age of MS symptom onset (years), mean (SD) & $33.5(10.3)$ \\
Time since symptom onset (years), mean (SD) & $14.3(9.9)$ \\
Sex, N (\%) & \\
$\quad$ Female & $438(77.8)$ \\
$\quad$ Male & $125(22.2)$
\end{tabular}

Race, N (\%)

White

Non-White

$523(96.1)$

$21(3.9)$

Education, N (\%)

High School or Lower

$149(27.1)$

College or Trade

University or Higher

$212(38.6)$

$188(34.2)$

Marital Status, N (\%)

Single

$71(12.8)$

Married/Common Law

Widowed/Divorced/Separated

$415(75.0)$

$67(12.1)$

Employment Status, N (\%)

Employed

$266(48.2)$

Unemployed

$286(51.8)$

Geographic Area, N (\%)

Calgary

$237(42.1)$

Edmonton

Central Alberta

Northern Alberta

Southern Alberta

Family History of MS, N (\%)

Yes

No

$176(31.3)$

83 (14.7)

$39(6.9)$

$28(5.0)$

$160(30.0)$

$373(70.0)$

Definite MS, N (\%)

Yes

No

Disease Course, N (\%)

RRMS

SPMS

PPMS

Uncertain

Disability Status (PDDS)

Normal

Mild

Moderate

Severe
Table 1 Demographic and MS characteristics of TAMSI sample (Continued)

\begin{tabular}{ll}
\hline Variable & Sample $(N=563)$ \\
\hline DMT Use, N (\%) & \\
Never & $178(32.1)$ \\
Past & $95(17.1)$ \\
Current & $281(50.7)$ \\
\hline
\end{tabular}

PDDS Patient Determined Disease Steps

DMT Disease-modifying therapy

${ }^{a}$ Note this category represents the 525 persons who indicated having definite MS

\section{Disability}

Scores on the HRQOL measures decreased with increasing disability on the PDDS, except for the MCS-36, where mean scores were similar across the disability levels (data not shown).

All HRQOL measures were able to distinguish between disability groups (PCS-36 F $=132.53, p<0.0001$; MCS$36 \mathrm{~F}=3.84, p=0.01$; SF-6D F $=45.18, p<0.0001$; HUI-III $\mathrm{F}=72.27, p<0.0001 ; \mathrm{PCS}-54 \mathrm{~F}=83.58, p<0.0001$; MCS$54 \mathrm{~F}=32.12, p<0.0001)$. Pairwise comparisons indicated that the PCS-36, SF-6D, HUI-III, and PCS-54 were able to distinguish between all disability groups (Table 3 ). The MCS-54 was able to distinguish between all groups except

Table 2 Comorbidities in TAMSI sample

\begin{tabular}{ll}
\hline Comorbidity & $\mathrm{N}(\%)$ \\
\hline Depression & $150(26.6)$ \\
Hypertension & $86(15.3)$ \\
Hyperlipidemia & $85(15.1)$ \\
Migraine & $84(14.9)$ \\
Lung Trouble & $49(8.7)$ \\
Thyroid Disease & $44(7.8)$ \\
Irritable Bowel Syndrome (IBS) & $42(7.5)$ \\
Osteoporosis & $42(7.5)$ \\
Anxiety Disorders & $38(6.7)$ \\
Cataracts & $24(4.3)$ \\
Diabetes & $20(3.6)$ \\
Heart Trouble & $14(2.5)$ \\
Inflammatory Bowel Disease (IBD) & $11(2.0)$ \\
Rheumatoid Arthritis & $11(2.0)$ \\
Fibromyalgia & $9(1.6)$ \\
Epilepsy & $7(1.2)$ \\
Bipolar Disorder & $6(1.1)$ \\
Glaucoma & $5(0.9)$ \\
Diseases of the Arteries & $4(0.7)$ \\
Lupus & $4(0.7)$ \\
Alcoholism & $3(0.5)$ \\
Parkinson's Disease & $0(0.0)$ \\
\hline
\end{tabular}




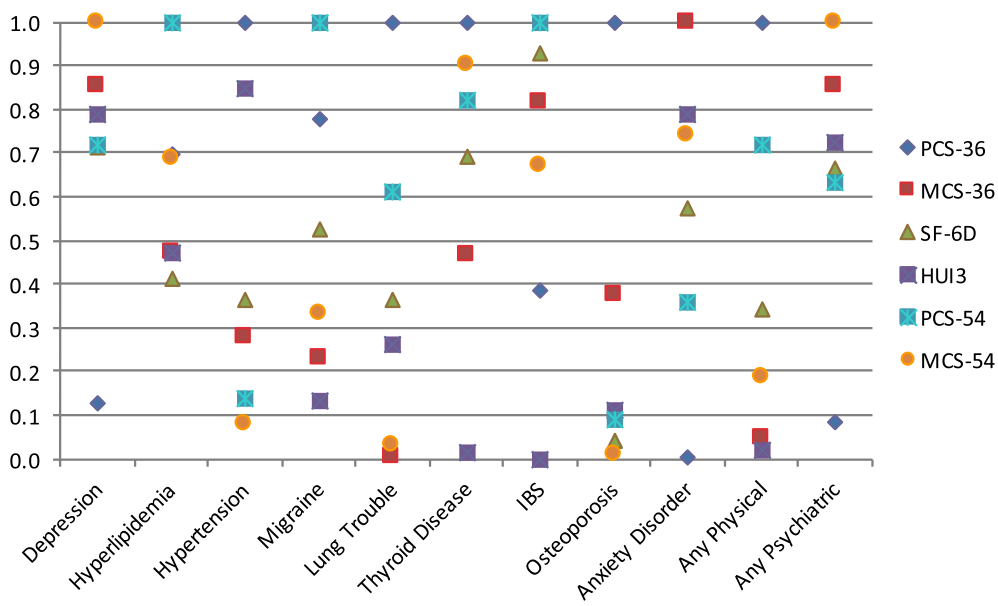

Fig. 2 Relative efficiency of HRQOL measures to discriminate absence/presence of comorbidities

persons with mild versus moderate disability. The MCS36 was only able to distinguish between persons with normal versus mild disability, and persons with mild versus moderate disability. Based on F-tests, the PCS-36 performed best overall; however the HUI-III was better able to distinguish between individuals with moderate versus severe disability. The RE of each instrument by disability level is displayed in Fig. 3.

\section{Discussion}

In this study, we evaluated the discriminative ability of multiple HRQOL measures to distinguish between persons with and without comorbidities in MS. We also examined the ability of these HRQOL scales to discriminate between varying levels of patient self-reported disability. The examined scales were only able to distinguish between persons with and without some comorbidities, particularly depression, and differed in their ability to distinguish individuals at higher levels of disability. The MCS-36 was not a good tool to distinguish between disability levels or number of comorbidities. The PCS-54 was slightly better than the generic tools at distinguishing between differing numbers of comorbidities, but it was comparable to the generic tools for levels of disability. In general, the MCS measures performed worse than the PCS measures for both the SF-36 and MSQOL-54, with the MCS-54 outperforming the MCS-36 for both comorbidity and disability discrimination. Of the utility measures, the SF-6D and HUIIII performed comparably for discriminating comorbidity and disability statuses.

In our study no measure was able to distinguish between those with no comorbidities and those with only one comorbidity, but the PCS-54 was very good at distinguishing between persons with different numbers of comorbidities, and the PCS-36, MCS-36, SF6D, HUI-III, and MCS-54 were also adequate. All HRQOL scales were very good at distinguishing persons with depression. Some HRQOL scales were also good at distinguishing those with hyperlipidemia and anxiety disorders. Overall the PCS-54 was slightly better at distinguishing between differing numbers of comorbidities than the generic tools. Of the utility measures, the SF-6D and HUI-III performed comparably for discriminating comorbidity statuses, although the SF-6D distinguished between persons with and

Table 3 Analysis of covariance for HRQOL measures by disability level*

\begin{tabular}{|c|c|c|c|c|c|c|c|c|c|c|c|c|}
\hline \multirow{2}{*}{$\begin{array}{l}\text { Dependent Variable } \\
\text { Independent Factor Variable }\end{array}$} & \multicolumn{2}{|l|}{ PCS-36 } & \multicolumn{2}{|c|}{ MCS-36 } & \multicolumn{2}{|l|}{ SF-6D } & \multicolumn{2}{|l|}{$\mathrm{HUI}-\mathrm{III}$} & \multicolumn{2}{|c|}{ PCS-54 } & \multicolumn{2}{|c|}{ MCS-54 } \\
\hline & F-test & $p$-value & F-test & p-value & F-test & $p$-value & F-test & $\mathrm{p}$-value & F-test & $p$-value & F-test & $p$-value \\
\hline Disability Status & 132.53 & $<0.0001$ & 3.84 & 0.01 & 45.18 & $<0.0001$ & 72.27 & $<0.0001$ & 83.58 & $<0.0001$ & 32.12 & $<0.0001$ \\
\hline Normal vs. Mild & 12.68 & $<0.05$ & 4.03 & $<0.05$ & 9.94 & $<0.05$ & 8.80 & $<0.05$ & 11.98 & $<0.05$ & 8.15 & $<0.05$ \\
\hline Normal vs. Moderate & 26.72 & $<0.05$ & 0.30 & NS & 13.69 & $<0.05$ & 15.68 & $<0.05$ & 19.71 & $<0.05$ & 10.82 & $<0.05$ \\
\hline Normal vs. Severe & 34.65 & $<0.05$ & 0.67 & NS & 20.58 & $<0.05$ & 26.93 & $<0.05$ & 26.47 & $<0.05$ & 16.98 & $<0.05$ \\
\hline Mild vs. Moderate & 14.04 & $<0.05$ & 4.33 & $<0.05$ & 3.75 & $<0.05$ & 6.88 & $<0.05$ & 7.73 & $<0.05$ & 2.67 & NS \\
\hline Mild vs. Severe & 21.96 & $<0.05$ & 3.36 & NS & 10.64 & $<0.05$ & 18.13 & $<0.05$ & 14.48 & $<0.05$ & 8.83 & $<0.05$ \\
\hline Moderate vs. Severe & 7.93 & $<0.05$ & 0.97 & NS & 6.90 & $<0.05$ & 11.25 & $<0.05$ & 6.75 & $<0.05$ & 6.16 & $<0.05$ \\
\hline
\end{tabular}

*Adjusted for age (continuous), sex, and disease course; BOLD type indicates $p<0.05$; NS not significant 


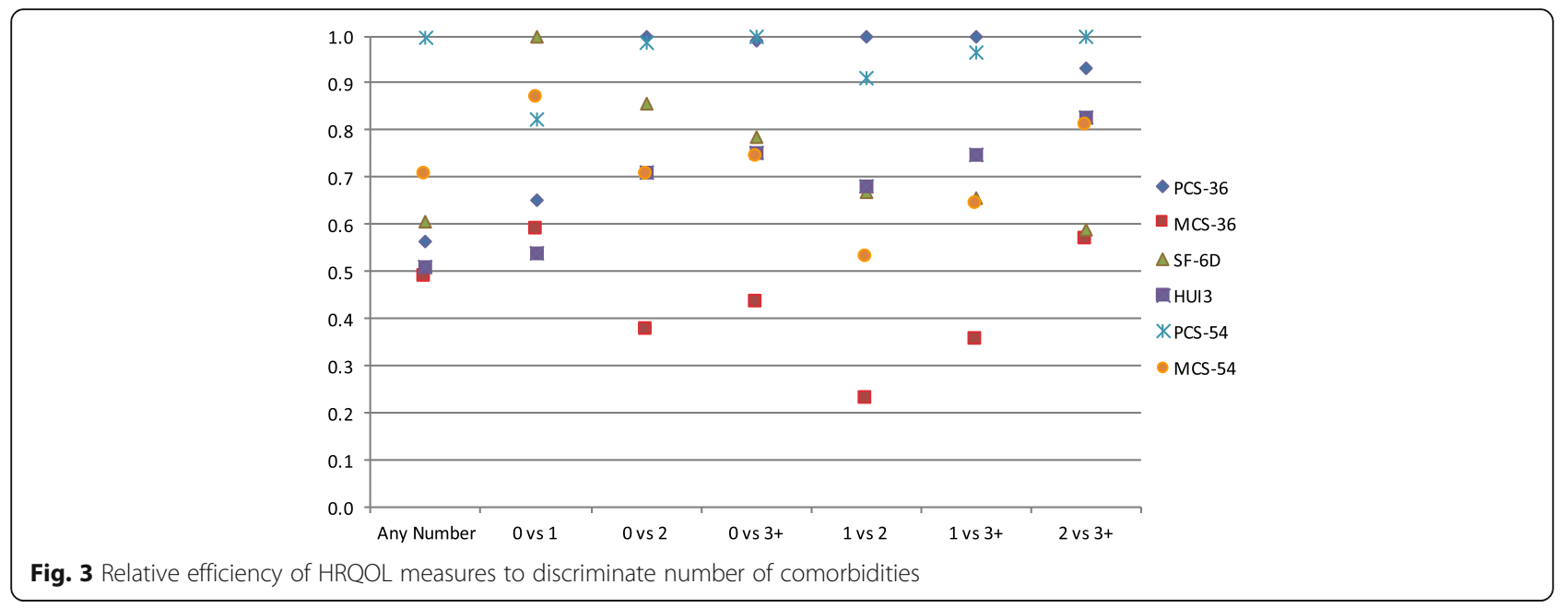

without any psychiatric comorbidity and between persons with and without migraine; whereas the HUI-III did not. It is possible to achieve more precision with the PCS-54 compared with the PCS-36, SF-6D or the HUI-III but this comes at the cost of additional questions (54 vs. 36, 11, 16). However, the length of the HRQOL tool does not appear to affect a subject's perception of the tool's utility [26]. Prior work has not identified clear differences in reliability and validity between disease-specific and general instruments when used cross-sectionally [26].

HRQOL scores were generally lowest in those persons with severe disability in the present study consistent with previous work [14]. There was a dose-response relationship between disability level and scores on all HRQOL measures, except the MSC-36. A prior study examining the utility of generic HRQOL measures across varying levels of MS-disability found that the HUI-III performed best to discriminate between all levels of disability [14]. As correlations between the MCS-54 and MCS-36 were very high, there may not be additional value in the extra 18 items on the MS-specific questionnaire (especially considering the amount of missing items on the optional sexual functioning subscale). We used a modified MSQOL-54 scoring algorithm to account for a large amount of missing data on the sexual function subscale. Missing data on this subscale is not uncommon in MS research [26], and other studies have employed modified algorithms to account for this issue [31]. We found the HUI-III was best able to discriminate between higher levels of disability (moderate and severe) as compared to the other generic and disease-specific measures. This is especially relevant in the context of progressive MS research and trials, where appropriate patient reported outcome measures must be carefully selected to ensure adequate sensitivity to change [32].
We examined generic and disease-specific health profile and utility measures of HRQOL in a large, welldescribed sample of persons with MS [33]. We explored the use of these tools across disability levels and comorbidity status, which has not been previously reported in the MS population. All tools except the SF-36 and MSQOL-54 are utility scales designed to put the effects of diverse illnesses onto a single scale; this is valuable when assessing patients with comorbidities. Limitations of the present study include the online nature of the survey, which may bias toward the inclusion of participants who are more educated, and with a higher income [33]. As noted previously, participants in this study were more likely to have a university education and to live in a neighborhood with a higher median income, compared to a reference MS population. The administration of the scales was not entirely independent, though the SF-36 was designed to be administered in this manner. This study was cross-sectional and therefore we did not examine the use of these tools over time; future studies should explore the responsiveness of these tools to change and individual response shift. Due to the online nature of the survey it was not possible for respondents to skip questions; as such scales were completed in their entirety or not at all, which may have resulted in more non-response on the HRQOL questionnaires. Whereas this study provided a broad-based comparison of two types of instruments, future studies seeking to compare specific instruments should consider adopting a priori criteria for assessing instrument performance.

\section{Conclusions}

Our findings suggest that future studies aimed at evaluating the effects of comorbidity or of comorbidity treatment on HRQOL in MS, may reasonably choose to employ generic or disease-specific measures depending on the MS population of interest. Disease-specific 
measures would discriminate better between those with and without comorbidities, and between those with differing numbers of comorbidities than generic measures. In contrast, the HUI-III would discriminate best between persons with moderate and severe disability. In studies in which the participants enrolled may have high levels of disability, the HUI-III appears best able to discriminate differences between participants, suggesting this may be a good measure for trials in progressive MS, although further evaluation is needed.

\section{Additional files}

Additional file 1: Table S1. Comparison of Health Related Quality of

Life Measures. (DOCX $12 \mathrm{~kb}$ )

Additional file 2: Figure S1. Mean score on HRQOL measures according to the number of comorbidities. (DOCX $29 \mathrm{~kb}$ )

Additional file 3: Table S2. Analysis of covariance for HRQOL measures by comorbidity status. (DOCX $14 \mathrm{~kb}$ )

\section{Abbreviations}

CCSVI: Chronic cerebrospinal venous insufficiency; EDSS: Expanded Disability Status Scale; HRQOL: Health Related Quality of Life; HUI: Health Utilities Index; IBD: Inflammatory bowel disease; IBS: Irritable bowel syndrome; MCS: Mental component score; MS: Multiple Sclerosis; MSQOL-54: Multiple Sclerosis Quality of Life-54; PCS: Physical component score; PDDS: Patient-Determined Disease Steps; RE: Relative efficiency; SF: Short Form; TAMSI: The Alberta Multiple Sclerosis Initiative

\section{Acknowledgements}

K. Fiest has received research funding from the Department of Critical Care Medicine, O'Brien Institute for Public Health and Calgary Centre for Clinical Research, all University of Calgary and the Critical Care Strategic Clinical Network, Alberta Health Services. S. Patten is the recipient of a salary support award (Senior Health Scholar) from Alberta Innovates, Health Solutions and receives research funding from $\mathrm{ClHR}$, the Institute of Health Economics and the Alberta Collaborative Research Grants Initiative. N. Jette is the holder of a Canada Research Chair in Neurological Health Services Research and received funding from: CIHR, Alberta Health, the Hotchkiss Brain Institute and the University of Calgary Cumming School of Medicine. R. A. Marrie receives research funding from: CIHR, Public Health Agency of Canada, Research Manitoba, Multiple Sclerosis Society of Canada, Multiple Sclerosis Scientific Foundation, Rx \& D Health Research Foundation, National Multiple Sclerosis Society, Crohn's and Colitis Canada, the Consortium of MS Centers, the Waugh Family Chair in Multiple Sclerosis.

\section{Funding}

This study was funded by a research grant from Alberta Health. The funding body had no role in the design of the study nor the collection, analysis, and interpretation of the data and in writing the manuscript.

\section{Availability of data and materials}

Please contact author for data requests.

\section{Authors' contributions}

All authors made contributions to the conception and design of this paper. $J G, L M, N J$, and RAM participated in the acquisition of data. JG conducted all data analyses. KF drafted the manuscript. All authors made substantial contributions to the interpretation of data and critically revised the manuscript for important intellectual content. All authors read and approved the final manuscript.

\section{Ethics approval and consent to participate}

Ethical approval was obtained from the University of Calgary's Conjoint Health Research Ethics Board and the University of Alberta's Health Research Ethics Board.
Consent for publication

Not applicable.

\section{Competing interests}

The authors declare they have no competing interests

\section{Publisher's Note}

Springer Nature remains neutral with regard to jurisdictional claims in published maps and institutional affiliations.

\section{Author details}

${ }^{1}$ Department of Internal Medicine, University of Manitoba, GF533, 820 Sherbrook Street, Winnipeg, MB R3A 1R9, Canada. ${ }^{2}$ Department of Clinical Neurosciences, University of Calgary, Calgary, AB, Canada. ${ }^{3}$ Hotchkiss Brain Institute, University of Calgary, Calgary, $\mathrm{AB}$, Canada. ${ }^{4}$ Department of Community Health Sciences, University of Calgary, Calgary, AB, Canada. ${ }^{5}$ Department of Psychiatry, University of Calgary, Calgary, AB, Canada. ${ }^{6}$ Mathison Centre for Mental Health Research and Education, University of Calgary, Calgary, AB, Canada. ${ }^{7} \mathrm{O}^{\prime}$ Brien Institute for Public Health, University of Calgary, Calgary, AB, Canada. ${ }^{8}$ Department of Community Health Sciences, University of Manitoba, Winnipeg, MB, Canada.

Received: 6 December 2015 Accepted: 14 December 2017 Published online: 21 December 2017

\section{References}

1. Marrie RA, Cohen J, Stuve O, Trojano M, Sorensen PS, Reingold S, et al. A systematic review of the incidence and prevalence of comorbidity in multiple sclerosis: overview. Mult Scler. 2015;21(3):263-81.

2. Marrie RA, Horwitz R, Cutter G, Tyry T, Campagnolo D, Vollmer T. Comorbidity, socioeconomic status and multiple sclerosis. Mult Scler. 2008; 14(8):1091-8.

3. Marrie RA, Horwitz R, Cutter G, Tyry T, Campagnolo D, Vollmer T. Comorbidity delays diagnosis and increases disability at diagnosis in MS. Neurology. 2009;72(2):117-24.

4. Weinstock-Guttman B, Zivadinov R, Mahfooz N, Carl E, Drake A, Schneider J, et al. Serum lipid profiles are associated with disability and MRI outcomes in multiple sclerosis. J Neuroinflammation. 2011;8:127.

5. Turpin KV, Carroll L, Cassidy JD, Hader WJ. Deterioration in the healthrelated quality of life of persons with multiple sclerosis: the possible warning signs. Mult Scler. 2007;13(8):1038-45.

6. Warren $\mathrm{S}$, Turpin $\mathrm{KV}$, Warren KG. Health-related quality of life in MS: issues and interventions. Can J Neurol Sci. 2009;36(5):540-1.

7. Berrigan L, Fisk J, Patten S, Tremlett H, Wolfson C, Warren S, et al. Healthrelated quality of life in multiple sclerosis: direct and indirect effects of comorbidity. Neurology. 2016;86(15):1417-24.

8. Benedict RH, Wahlig E, Bakshi R, Fishman I, Munschauer F, Zivadinov R, et al. Predicting quality of life in multiple sclerosis: accounting for physical disability, fatigue, cognition, mood disorder, personality, and behavior change. J Neurol Sci. 2005;231(1-2):29-34.

9. Nortvedt MW, Riise T, Myhr KM, Nyland HI. Quality of life in multiple sclerosis: measuring the disease effects more broadly. Neurology. 1999 ;53(5):1098-103.

10. Kobelt $G$, Berg J, Lindgren $P$, Fredrikson $S$, Jonsson B. Costs and quality of life of patients with multiple sclerosis in Europe. J Neurol Neurosurg Psychiatry. 2006;77(8):918-26

11. Motl RW, Gosney JL. Effect of exercise training on quality of life in multiple sclerosis: a meta-analysis. Mult Scler. 2008;14(1):129-35.

12. Zwibel HL. Contribution of impaired mobility and general symptoms to the burden of multiple sclerosis. Adv Ther. 2009;26(12):1043-57.

13. Grima DT, Torrance GW, Francis G, Rice G, Rosner AJ, Lafortune L. Cost and health related quality of life consequences of multiple sclerosis. Mult Scler. 2000:6(2):91-8.

14. Fisk JD, Brown MG, Sketris IS, Metz LM, Murray TJ, Stadnyk KJ. A comparison of health utility measures for the evaluation of multiple sclerosis treatments. J Neurol Neurosurg Psychiatry. 2005;76(1):58-63.

15. Hohol MJ, Orav EJ, Weiner HL. Disease steps in multiple sclerosis: a simple approach to evaluate disease progression. Neurology. 1995;45(2):251-5.

16. Learmonth YC, Motl RW, Sandroff BM, Pula JH, Cadavid D. Validation of patient determined disease steps (PDDS) scale scores in persons with multiple sclerosis. BMC Neurol. 2013;13:37. 
17. Wiebe S, Guyatt G, Weaver B, Matijevic S, Sidwell C. Comparative responsiveness of generic and specific quality-of-life instruments. J Clin Epidemiol. 2003;56(1):52-60.

18. Horsman J, Furlong W, Feeny D, Torrance G. The health utilities index (HUI): concepts, measurement properties and applications. Health Qual Life Outcomes. 2003;1:54.

19. Vickrey BG, Hays RD, Harooni R, Myers LW, Ellison GW. A health-related quality of life measure for multiple sclerosis. Qual Life Res. 1995;4(3): 187-206.

20. Ware JE Jr, Sherbourne CD. The MOS 36-item short-form health survey (SF-36). I. Conceptual framework and item selection. Med Care. 1992; 30(6):473-83.

21. Brazier J, Roberts J, Deverill M. The estimation of a preference-based measure of health from the SF-36. J Health Econ. 2002;21(2):271-92.

22. Dallmeijer AJ, de Groot V, Roorda LD, Schepers VP, Lindeman E, van den Berg LH, et al. Cross-diagnostic validity of the SF-36 physical functioning scale in patients with stroke, multiple sclerosis and amyotrophic lateral sclerosis: a study using Rasch analysis. J Rehabil Med. 2007;39(2):163-9.

23. Riazi A, Hobart JC, Lamping DL, Fitzpatrick R, Thompson AJ. Evidence-based measurement in multiple sclerosis: the psychometric properties of the physical and psychological dimensions of three quality of life rating scales. Mult Scler. 2003:9(4):411-9.

24. Kuspinar A, Mayo NE. A review of the psychometric properties of generic utility measures in multiple sclerosis. PharmacoEconomics. 2014; 32(8):759-73.

25. Freeman JA, Hobart JC, Thompson AJ. Does adding MS-specific items to a generic measure (the SF-36) improve measurement? Neurology. 2001:57(1):68-74.

26. Moore F, Vickrey B, Fortin K, Lee L. Two multiple sclerosis quality-of-life measures: comparison in a National Sample. Can J Neurol Sci. 2015; 42(1):55-63.

27. Horton M, Rudick RA, Hara-Cleaver C, Marrie RA. Validation of a self-report comorbidity questionnaire for multiple sclerosis. Neuroepidemiology. 2010 35(2):83-90

28. Swinscow TDV, 11. Correlation and regression, in Statistics at Square One. London: BMJ Publishing Group. 1997.

29. Luo N, Wang P, AZ F, Johnson JA, Coons SJ. Prefderence-based SF-6D scores derived from the SF-36 and SF-12 have different discriminative power in a population health survey. Med Care. 2012;50(7):627-32.

30. Fiest KM, Fisk JD, Patten SB, Tremlett H, Wolfson C, Warren S, et al, Comorbidity is associated with pain-related activity limitations in multiple sclerosis. Mult Scler Relat Disord. 2015:4(5):470-6.

31. Buhse M, Banker WM, Clement LM. Factors associated with health-related quality of life among older people with multiple sclerosis. Int J MS Care. 2014;16(1):10-9.

32. Ontaneda D, Fox RJ, Chataway J. Clinical trials in progressive multiple sclerosis: lessons learned and future perspectives. Lancet Neurol. 2015; 14(2):208-23.

33. Metz LM, Greenfield J, Marrie R, Jette N, Blevens G, Svenson L, et al. Medical tourism for CCSVI procedures in people with MS: an observational study. Can J Neurol Sci. 2016;43(3):360-7.

\section{Submit your next manuscript to BioMed Central and we will help you at every step:}

- We accept pre-submission inquiries

- Our selector tool helps you to find the most relevant journal

- We provide round the clock customer support

- Convenient online submission

- Thorough peer review

- Inclusion in PubMed and all major indexing services

- Maximum visibility for your research

Submit your manuscript at www.biomedcentral.com/submit
Biomed Central 